\title{
An Improved Matrix Perturbation Method for Modified
}

\section{Structures}

\section{Zhong-hai $\mathrm{XU}^{1, *}$, Zhong-yuan $\mathrm{NIU}^{2}$, Jian-zhao ZHANG ${ }^{3}$ and}

\author{
Rong-guo WANG ${ }^{4}$ \\ ${ }^{1,2,3,4}$ National Key Laboratory of Science and Technology on Advanced Composites in \\ Special Environments \\ Harbin Institute of Technology, Harbin, 150080, PR China. \\ *xuzh@hit.edu.cn
}

Keywords: Matrix perturbation method, Iterative, Eigensolution, Natural frequency, Rayleigh quotient.

\begin{abstract}
In this paper, an improved matrix perturbation method for modified structures is presented. First we construct an iterative format on the basis of the matrix perturbation theory. By introducing a pair of initial eigensolution, we can obtain the natural vibration mode of the modified structure, and then use the Rayleigh quotient to compute the corresponding natural frequency. In this way, the computation accuracy can be greatly improved. Finally, a numerical example is included to demonstrate the validity of the proposed method.
\end{abstract}

\section{Introduction}

Structural dynamic reanalysis play an important role in structural design and optimization. Many scholars have paid their attentions to this subject.

The traditional method is the Taylor series expansion method, and later approximation method using reduced basis vectors was proposed. In the papers of Noorland Nair et al.2, the basis vector is the Taylor series expansion of the eigenpairs with respect to the structural parameters, and in the papers of Aktas and Moses3and Kirsch4, the basis vector is the binomial expansion by solving a static problem. However, the choice of the basis vector is still a research issue. Kirsch5,6,7introduced the combined approximation algorithm to the vibration analysis process, and made the equivalent treatment for the modal equations and the static equilibrium equations, then constructed the reduced basis vector in the Krylov subspace, finally, gave the error evaluation and solving process. He et al.8made the modal reanalysis research for structural large topological modifications with added degrees of freedom. Rong et al.9, Ma et al.10, Yang et al.11 and Chen et al.12 proposed the extended combined approximation method, and improved the calculation accuracy using the Rayleigh quotient. Zhang et al.13presented the modified combined approximation method based on the inverse iteration and combined approximation algorithm, and improved the calculation accuracy for structural large modifications. Liu et al.14made the 
research on the eigenvalue repeated analysis problems. Chen et al.15,16made the research on the structural dynamic responds repeated analysis problems by introducing the Epsilon algorithm and combining with the Newmann series expansion.

Though many algorithms above are involved, the matrix perturbation method is the classical approach. Yang et al.17proposed a method based on the Pade approximation to improve the perturbation theory. Chen et al.18improved the calculation accuracy of the perturbation method by combining the matrix perturbation method with the Rayleigh quotient, but it isn't usually applicable to the case of large parameter modifications, as noted in the paper of Chen et al.19. So it is necessary to improve the perturbation method in the calculation accuracy and the range of application.

In this paper, we present an improved matrix perturbation method. By combining the iteration algorithm with the Rayleigh quotient, we can obtain the eigensolution of the modified structure, and the computational accuracy can be greatly improved. Numerical example is used to illustrate the validity of the proposed method.

\section{Theoretical Background}

Consider the following general structural vibration eigenvalue problem

$$
\begin{aligned}
& {[K]\left\{u_{i}\right\}=\lambda_{i}[M]\left\{u_{i}\right\} .} \\
& \left\{u_{i}\right\}^{T}[M]\left\{u_{j}\right\}=\delta_{i j}, \quad i, j=1,2, \ldots n .
\end{aligned}
$$

where $[K]$ and $[M]$ are the structural stiffness and mass matrices, respectively, $\lambda_{i}=w_{i}^{2}$ is the ith eigenvalue, $w_{i}$ is the ith natural frequency, $\left\{u_{i}\right\}$ is the eigenvector corresponding to $\lambda_{i}, n$ is the total degrees of freedom, and $\delta_{i j}$ is the Kronecker sign. It is assumed that the eigenvalue is distinct, and Eq. (1) will be referred to as the original eigenvalue problem.

After the structure is modified, the stiffness matrix $[K]$ and the mass matrix $[M]$ are also changed, and the amounts of change are $[\Delta K]$ and $[\Delta M]$, respectively. The corresponding eigenvalue problem of the modified structure is

$$
\begin{aligned}
& {[\bar{K}]\left\{\bar{u}_{i}\right\}=\bar{\lambda}_{i}[\bar{M}]\left\{\bar{u}_{i}\right\} .} \\
& \left\{\bar{u}_{i}\right\}^{T}[\bar{M}]\left\{\bar{u}_{j}\right\}=\delta_{i j}, \quad i, j=1,2, \ldots n .
\end{aligned}
$$

where 


$$
\begin{aligned}
& {[\bar{K}]=[K]+[\Delta K] .} \\
& {[\bar{M}]=[M]+[\Delta M] .}
\end{aligned}
$$

Then the ith eigenvalue $\bar{\lambda}_{i}$ and the corresponding eigenvector $\left\{\bar{u}_{i}\right\}$ can be expressed as

$$
\bar{\lambda}_{i}=\lambda_{i}+\Delta \lambda_{i}, \quad\left\{\bar{u}_{i}\right\}=\left\{u_{i}\right\}+\left\{\Delta u_{i}\right\}
$$

\section{The First-order Perturbation Method}

From Eqs. (6), the standard equations of the first-order perturbation method can be written as

$$
\lambda_{1 i}=\lambda_{i}+\Delta \lambda_{1 i}, \quad\left\{u_{1 i}\right\}=\left\{u_{i}\right\}+\left\{\Delta u_{1 i}\right\} .
$$

where

$$
\Delta \lambda_{1 i}=\left\{u_{i}\right\}^{T}\left([\Delta K]-\lambda_{i}[\Delta M]\right)\left\{u_{i}\right\}, \quad\left\{\Delta u_{1 i}\right\}=\sum_{j=1}^{n} c_{i j}^{1}\left\{u_{j}\right\}
$$

In the above formula, coefficients $c_{i j}^{1}$ are given as follows

$$
\begin{gathered}
c_{i j}^{1}=\left\{u_{j}\right\}^{T}\left([\Delta K]-\lambda_{i}[\Delta M]\right)\left\{u_{i}\right\} /\left(\lambda_{i}-\lambda_{j}\right), \quad j \neq i . \\
c_{i i}^{1}=-\left\{u_{i}\right\}^{T}[\Delta M]\left\{u_{i}\right\} / 2, \quad j=i .
\end{gathered}
$$

\section{The second-order Perturbation Method}

Similarly, the standard equations of the second-order perturbation method can be written as

$$
\lambda_{2 i}=\lambda_{i}+\Delta \lambda_{2 i}, \quad\left\{u_{2 i}\right\}=\left\{u_{i}\right\}+\left\{\Delta u_{2 i}\right\}
$$

where

$$
\begin{aligned}
& \Delta \lambda_{2 i}=\Delta \lambda_{1 i}+\left\{u_{i}\right\}^{T}[\Delta K]\left\{\Delta u_{1 i}\right\}-\lambda_{i}\left\{u_{i}\right\}^{T}[\Delta M]\left\{\Delta u_{1 i}\right\} \\
& -\Delta \lambda_{1 i}\left\{u_{i}\right\}^{T}[M]\left\{\Delta u_{1 i}\right\}-\Delta \lambda_{1 i}\left\{u_{i}\right\}^{T}[\Delta M]\left\{u_{i}\right\} \\
& \left\{\Delta u_{2 i}\right\}=\left\{\Delta u_{1 i}\right\}+\sum_{j=1}^{n} c_{i j}^{2}\left\{u_{j}\right\}
\end{aligned}
$$


In the above formula, coefficients $c_{i j}^{2}$ are given as follows

$$
\begin{aligned}
& c_{i j}^{2}=\left(\left\{u_{j}\right\}^{T}[\Delta K]\left\{\Delta u_{1 i}\right\}-\lambda_{i}\left\{u_{j}\right\}^{T}[\Delta M]\left\{\Delta u_{1 i}\right\}\right. \\
& \left.-\Delta \lambda_{1 i}\left\{u_{j}\right\}^{T}[M]\left\{\Delta u_{1 i}\right\}-\Delta \lambda_{1 i}\left\{u_{j}\right\}^{T}[\Delta M]\left\{u_{i}\right\}\right) /\left(\lambda_{i}-\lambda_{j}\right), j \neq i . \\
& c_{i i}^{2}=-\left(\left\{\Delta u_{1 i}\right\}^{T}[M]\left\{\Delta u_{1 i}\right\}+\left\{u_{i}\right\}^{T}[\Delta M]\left\{\Delta u_{1 i}\right\}+\left\{\Delta u_{1 i}\right\}^{T}[\Delta M]\left\{u_{i}\right\}\right) / 2 \quad j=i .
\end{aligned}
$$

\section{William B.B. Method Mentioned by William20}

Use the inner product of eigenvector with respect to the modified structure mass matrix $\bar{M}$ to replace the inner product with respect to the original structure mass matrix $M$, we can obtain the William.B.B method.

Similarly, the standard equations of the William.B.B method can be written as

$$
\lambda_{3 i}=\lambda_{i}+\Delta \lambda_{3 i}, \quad\left\{u_{3 i}\right\}=\left\{u_{i}\right\}+\left\{\Delta u_{3 i}\right\} .
$$

where

$$
\begin{aligned}
& \Delta \lambda_{3 i}=\left\{u_{i}\right\}^{T}\left([\Delta K]-\lambda_{i}[\Delta M]\right)\left\{u_{i}\right\} /\left(\left\{u_{i}\right\}^{T}[\bar{M}]\left\{u_{i}\right\}\right) . \\
& \left\{\Delta u_{3 i}\right\}=\sum_{j=1}^{n} c_{i j}^{3}\left\{u_{j}\right\}
\end{aligned}
$$

In the above formula, coefficients $c^{3}$ are given as follows

$$
\begin{aligned}
& c_{i j}^{3}=\left\{u_{j}\right\}^{T}\left([\Delta K]-\lambda_{i}[\Delta M]\right)\left\{u_{i}\right\} /\left(\left(\lambda_{i}-\lambda_{j}\right)\left\{u_{i}\right\}^{T}[\bar{M}]\left\{u_{i}\right\}\right), j \neq i \\
& c_{i i}^{3}=-\left\{u_{i}\right\}^{T}[\Delta M]\left\{u_{i}\right\} /\left(2\left\{u_{i}\right\}^{T}[\bar{M}]\left\{u_{i}\right\}\right), j=i .
\end{aligned}
$$

The Perturbation Method Combining with the Rayleigh Quotient Mentioned by Chen Et Al.21

Similarly, the standard equations can be written as

$$
\lambda_{4 i}=\lambda_{i}+\Delta \lambda_{4 i}, \quad\left\{u_{4 i}\right\}=\left\{u_{i}\right\}+\left\{\Delta u_{4 i}\right\} .
$$

Consider the effects of the high-frequency modes and use the first-order perturbation method results, then have

$$
\Delta \lambda_{4 i}=\left\{u_{1 i}\right\}^{T}\left([\bar{K}]-\lambda_{i}[\bar{M}]\right)\left\{u_{1 i}\right\} /\left(\left\{u_{1 i}\right\}^{T}[\bar{M}]\left\{u_{1 i}\right\}\right)
$$




$$
\left\{\Delta u_{4 i}\right\}=\sum_{j=1}^{n} c_{i j}^{4}\left\{u_{j}\right\}
$$

In the above formula, coefficients $c_{i j}^{4}$ are given as follows:

$$
\begin{aligned}
& c_{i j}^{4}=\left\{u_{j}\right\}^{T}\left([\Delta K]-\lambda_{i}[\Delta M]-\Delta \lambda_{1 i}[\bar{M}]\right)\left\{u_{1 i}\right\} /\left(\lambda_{i}-\lambda_{j}\right), j \neq i . \\
& c_{i i}^{4}=-\left(\left\{u_{1 i}\right\}^{T}[\Delta M]\left\{u_{1 i}\right\}+\left\{\Delta u_{1 i}\right\}^{T}[M]\left\{\Delta u_{1 i}\right\}\right) / 2, j=i .
\end{aligned}
$$

\section{The Proposed Method}

From the previous four methods, we know that we first calculate the eigenvalues of the modified structure, and then calculate the corresponding eigenvectors. The following we first construct an iteration format on the basis of the matrix perturbation theory to compute the natural vibration mode of the modified structure, and then use the Rayleigh quotient to compute the corresponding natural frequency.

The eigenvectors of modified structure are expressed as

$$
\begin{gathered}
\left\{u_{n i}^{k+1}\right\}=\left\{u_{\mathrm{i}}\right\}+\left\{\Delta u_{n i}^{k+1}\right\} . \\
\left\{\Delta u_{n i}^{k+1}\right\}=\sum_{j=1}^{n} c_{i j}^{n(k+1)}\left\{u_{j}\right\}
\end{gathered}
$$

Substituting Eqs. (5)-(6) into Eq. (3) and rearranging them yields

$$
\begin{aligned}
& -\left([K]-\lambda_{i}[M]\right)\left\{\Delta u_{i}\right\}= \\
& \left([\Delta K]-\lambda_{i}[\Delta M]\right)\left(\left\{u_{i}\right\}+\left\{\Delta u_{i}\right\}\right)-\Delta \lambda_{i}([M]+[\Delta M])\left(\left\{u_{i}\right\}+\left\{\Delta u_{i}\right\}\right) .
\end{aligned}
$$

Let $\Delta \lambda_{i}=\Delta \lambda_{n i}^{k},\left\{\Delta u_{i}\right\}=\left\{\Delta u_{n i}^{k}\right\}$ on Eq. (28) right-hand side, and we get

$$
-\left([K]-\lambda_{i}[M]\right)\left\{\Delta u_{i}\right\}=\left([\Delta K]-\lambda_{i}[\Delta M]\right)\left\{u_{n i}^{k}\right\}-\Delta \lambda_{n i}^{k}[\bar{M}]\left\{u_{n i}^{k}\right\} .
$$

Premultiplying Eq. (29) by $\left\{u_{j}\right\}^{T}$ results in

$$
\begin{aligned}
& -\left\{u_{j}\right\}^{T}\left([K]-\lambda_{i}[M]\right)\left\{\Delta u_{i}\right\}= \\
& \left\{u_{j}\right\}^{T}\left([\Delta K]-\lambda_{i}[\Delta M]\right)\left\{u_{n i}^{k}\right\}-\Delta \lambda_{n i}^{k}\left\{u_{j}\right\}^{T}[\bar{M}]\left\{u_{n i}^{k}\right\} .
\end{aligned}
$$

In Eq. (30), let $\left\{\Delta u_{i}\right\}=\left\{\Delta u_{n i}^{k+1}\right\}$. Substituting Eq. (27) into Eq. (30) and noting that $\left\{u_{j}\right\}$ is M-normalized, we have the following iterative formula 


$$
c_{i j}^{n(k+1)}=\left\{u_{j}\right\}^{T}\left([\Delta K]-\lambda_{i}[\Delta M]-\Delta \lambda_{n i}^{k} \bar{M}\right)\left\{u_{n i}^{k}\right\} /\left(\lambda_{i}-\lambda_{j}\right) \quad j \neq i .
$$

For the case of $j=i$, the coefficient $c_{i i}^{n(k+1)}$ can be obtained by the $\bar{M}$-normalized condition

$$
\left(\left\{u_{i}\right\}+\left\{\Delta u_{i}\right\}\right)^{T}[\bar{M}]\left(\left\{u_{i}\right\}+\left\{\Delta u_{i}\right\}\right)=1 .
$$

Expanding Eq. (32) and rearranging them yields

$$
-2\left\{u_{i}\right\}^{T}[M]\left\{\Delta u_{i}\right\}=\left(\left\{u_{i}\right\}+\left\{\Delta u_{i}\right\}\right)^{T}[\Delta M]\left(\left\{u_{i}\right\}+\left\{\Delta u_{i}\right\}\right)+\left\{\Delta u_{i}\right\}^{T}[M]\left\{\Delta u_{i}\right\}
$$

Similarly, noting Eqs. (2) and (27), we can obtain

$$
c_{i i}^{n(k+1)}=-\left(\left\{u_{n i}^{k}\right\}^{T}[\Delta M]\left\{u_{n i}^{k}\right\}+\left\{\Delta u_{n i}^{k}\right\}[M]\left\{\Delta u_{n i}^{k}\right\}\right) / 2 .
$$

Based on the obtained $c_{i i}^{n(k+1)}$ and $c_{i j}^{n(k+1)}$, the eigenvectors $\left\{u_{n i}^{k+1}\right\}$ can be obtained from Eqs. (26) and (27).

Then we use the Rayleigh quotient and get the eigenvalues

$$
\lambda_{n i}^{k+1}=\left(\left\{u_{n i}^{k+1}\right\}^{T}[\bar{K}]\left\{u_{n i}^{k+1}\right\}\right) /\left(\left\{u_{n i}^{k+1}\right\}^{T}[\bar{M}]\left\{u_{n i}^{k+1}\right\}\right) .
$$

and further get

$$
\Delta \lambda_{n i}^{k+1}=\left\{u_{n i}^{k+1}\right\}^{T}\left([\bar{K}]-\lambda_{i}[\bar{M}]\right)\left\{u_{n i}^{k+1}\right\} /\left(\left\{u_{n i}^{k+1}\right\}^{T}[\bar{M}]\left\{u_{n i}^{k+1}\right\}\right) .
$$

Throughout the iterative process, we first select the iteration-based initial values $\Delta \lambda_{n i}^{0},\left\{\Delta u_{n i}^{0}\right\}$ and $\left\{u_{n i}^{0}\right\}$. After obtaining the $\Delta \lambda_{n i}^{k+1},\left\{\Delta u_{n i}^{k+1}\right\}$ and $\left\{u_{n i}^{k+1}\right\}$, we regard them as the new iteration-based and substitute them into Eqs. (31) and (34), and make the repeated iteration of the loop equation until the results converge to the required accuracy.

\section{Numerical Example}

In this section, we will use a numerical example to demonstrate the validity of the proposed method.

We consider the following truss with 8 joints which is shown in Fig. 1. The cross section of all members is $A=1.2 \times 10^{-3} \mathrm{~m}^{2}$, the modulus of elasticity is $E=2.1 \times 10^{11} \mathrm{~Pa}$, and the density is $\rho=7.85 \times 10^{3} \mathrm{~kg} / \mathrm{m}^{3}$. We will adopt the lumped mass in the finite element computation. We assume that the cross section of the bar (1) is increased by $20 \%$ and $60 \%$, respectively. 
To compare the accuracies, we adopt the first-order perturbation method, the second-order perturbation method, the William.B.B method, the perturbation method combining with the Rayleigh quotient and the proposed method respectively to compute the eigenvalues and eigenvectors of the modified structure. In the proposed method, we choose the results of first-order perturbation method as the initial iteration base, and make iteration one time, which makes the cost of computing minimum. For convenience, we denote the above five methods as method 1-method 5 sequentially in the following Figures.

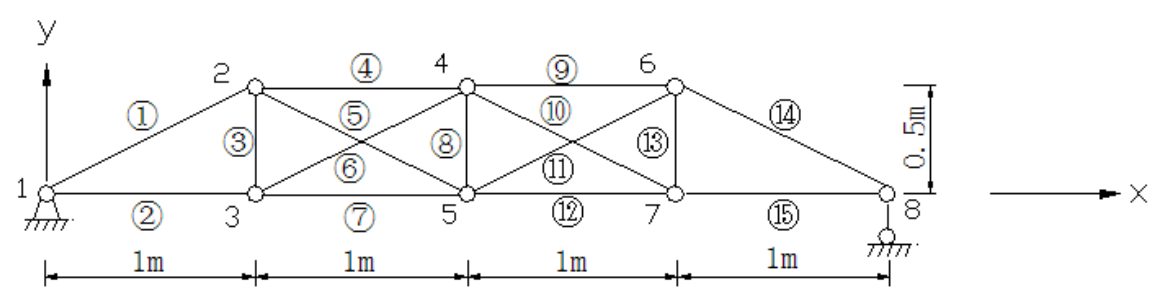

Fig. 1 The truss with 8 joints

The error of eigenvalue is calculated by

$t r_{i}=\left|\left(\lambda_{E i}-\lambda_{I i}\right) / \lambda_{E i}\right|$.

$\lambda_{E i}$ is the exact eigenvalue of the modified structure, $\lambda_{I i}$ is the approximate eigenvalue computed by the previous several methods. The errors are denoted by $\operatorname{tr} 1$, $\operatorname{tr} 2, \operatorname{tr} 3, \operatorname{tr} 4$ and trn 1 , respectively.

The error of eigenvector is calculated by

$$
u r_{i}=\operatorname{abs}\left(\left\{u_{E i}\right\}^{T} *\left\{u_{I i}\right\} / \operatorname{sqrt}\left(\left\{u_{E i}\right\}^{T} *\left\{u_{E i}\right\}\right) / \operatorname{sqrt}\left(\left\{u_{I i}\right\}^{T} *\left\{u_{I i}\right\}\right)\right)
$$

$u_{E i}$ is the exact eigenvector of the modified structure, $u_{I i}$ is the approximate eigenvector computed by the previous several methods. The errors are denoted by ur1, ur2, ur3, ur4 and urn1, respectively.

The error results of the first five eigensolutions are listed in Tables 1-4. We use the first-order perturbation solution as the initial iteration base, and make iteration one time in the proposed method, at this time Eqs. (31) and (34) are degenerated into Eqs. (24) and (25), respectively, so Eq. (26) is identical to Eq. (21), as we have seen in Tables 3-4, the values of urn1 and ur4 are the same, i.e. the eigenvector accuracy of the proposed method is consistent with that obtained by the perturbation method combining with the Rayleigh quotient, but more accurate than the other three methods, as we have seen in Figs. 4-5. The eigenvalue accuracy of the proposed method is much higher than the other four methods, as shown in Tables 1-2 and Figs. 2-3. We also know when a large amount of structure modification, such as modification of $60 \%$, as shown in Tables 2 and 4, Figs. 3 and 5, the proposed method also has high accuracy, and the validity of the proposed method is demonstrated. 
Table 1 The eigenvalue accuracies comparison (cross section is increased by $20 \%$ )

\begin{tabular}{cccccc}
\hline $\mathrm{i}$ & $\operatorname{tr} 1$ & $\operatorname{tr} 2$ & $\operatorname{tr} 3$ & $\operatorname{tr} 4$ & $\operatorname{trn} 1$ \\
\hline 1 & 0.0016 & 0.0003 & 0.0015 & $5.57 \mathrm{e}-5$ & $1.91 \mathrm{e}-6$ \\
2 & 0.0004 & 0.0001 & 0.0004 & $4.32 \mathrm{e}-5$ & $3.84 \mathrm{e}-6$ \\
3 & 0.0040 & 0.0003 & 0.0037 & $1.54 \mathrm{e}-5$ & $1.37 \mathrm{e}-6$ \\
4 & 0.0003 & $4.4 \mathrm{e}-5$ & 0.0002 & $3.04 \mathrm{e}-6$ & $2.96 \mathrm{e}-8$ \\
5 & $7.81 \mathrm{e}-5$ & $4.75 \mathrm{e}-7$ & $1.08 \mathrm{e}-5$ & $1.93 \mathrm{e}-7$ & $1.64 \mathrm{e}-10$ \\
\hline
\end{tabular}

Table 2 The eigenvalue accuracies comparison (cross section is increased by $60 \%$ )

\begin{tabular}{cccccc}
\hline $\mathrm{i}$ & $\operatorname{tr} 1$ & $\operatorname{tr} 2$ & $\operatorname{tr} 3$ & $\operatorname{tr} 4$ & $\operatorname{trn} 1$ \\
\hline 1 & 0.0107 & 0.0061 & 0.0102 & 0.0034 & 0.0011 \\
2 & 0.0024 & 0.0027 & 0.0026 & 0.0024 & 0.0020 \\
3 & 0.0275 & 0.0075 & 0.0257 & 0.0014 & 0.0005 \\
4 & 0.0031 & 0.0009 & 0.0022 & 0.0002 & $2.01 \mathrm{e}-5$ \\
5 & 0.0007 & $6.48 \mathrm{e}-6$ & 0.0001 & $1.42 \mathrm{e}-5$ & $1.08 \mathrm{e}-7$ \\
\hline
\end{tabular}

Table 3 The eigenvector accuracies comparison (cross section is increased by $20 \%$ )

\begin{tabular}{cccccc}
\hline $\mathrm{i}$ & ur1 & ur2 & ur3 & ur4 & urn1 \\
\hline 1 & 0.999998 & 0.999999 & 0.999998 & 1.000000 & 1.000000 \\
2 & 0.999964 & 0.999997 & 0.999967 & 0.999997 & 0.999997 \\
3 & 0.999961 & 0.999997 & 0.999964 & 0.999997 & 0.999997 \\
4 & 0.999993 & 1.000000 & 0.999995 & 1.000000 & 1.000000 \\
5 & 1.000000 & 1.000000 & 1.000000 & 1.000000 & 1.000000 \\
\hline
\end{tabular}

Table 4 The eigenvector accuracies comparison (cross section is increased by $60 \%$ )

\begin{tabular}{cccccc}
\hline $\mathrm{i}$ & ur1 & ur2 & ur3 & ur4 & urn1 \\
\hline 1 & 0.999885 & 0.999964 & 0.999899 & 0.999965 & 0.999965 \\
2 & 0.998633 & 0.998883 & 0.998781 & 0.998879 & 0.998879 \\
3 & 0.998357 & 0.998924 & 0.998543 & 0.998937 & 0.998937 \\
4 & 0.999511 & 0.999992 & 0.999646 & 0.999992 & 0.999992 \\
5 & 0.999973 & 1.000000 & 0.999991 & 1.000000 & 1.000000 \\
\hline
\end{tabular}

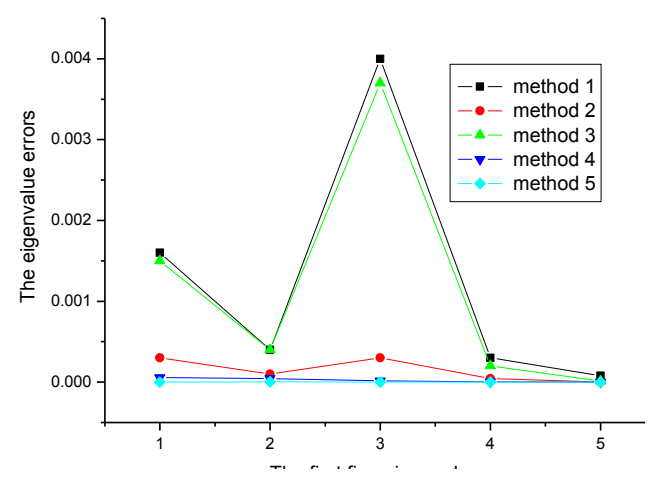

Fig. 2 The eigenvalue accuracies comparison (cross section is increased by $20 \%$ ) 


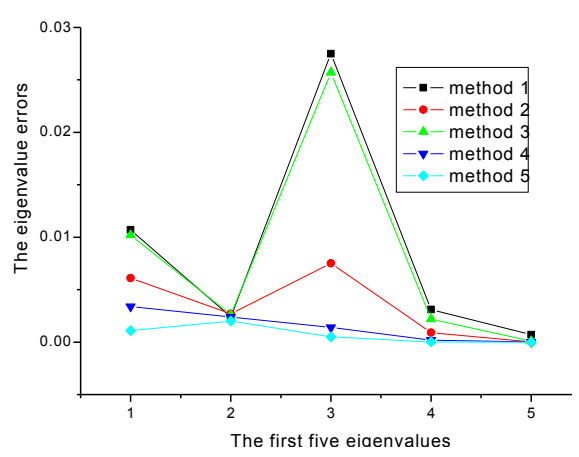

Fig. 3 The eigenvalue accuracies comparison (cross section is increased by $60 \%$ )

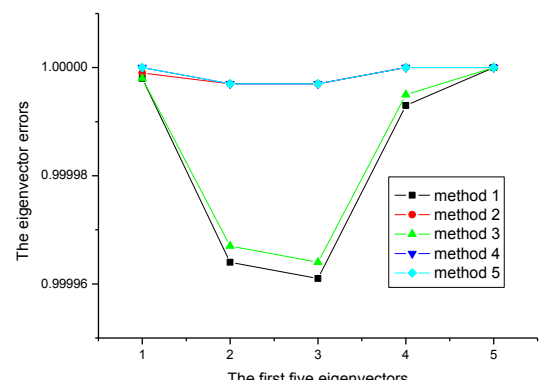

Fig.4 The eigenvector accuracies comparison (cross section is increased by $20 \%$ )

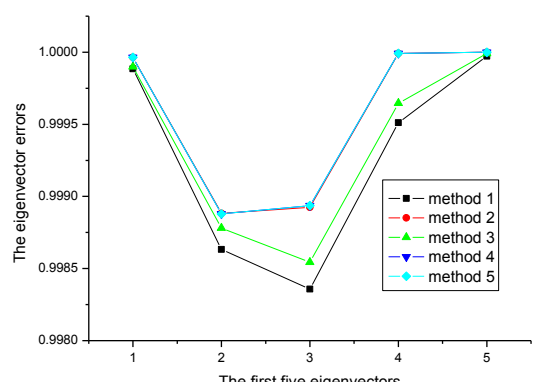

Fig. 5 The eigenvector accuracies comparison (cross section is increased by $60 \%$ )

\section{Conclusions}

An improved matrix perturbation method for modified structures has been proposed. By constructing an iterative format and combining with the Rayleigh quotient, we can obtain the eigensolution of the modified structure, and the calculation accuracy can be greatly improved. A numerical example has demonstrated the validity of the proposed method. 


\section{Acknowledgments}

The work was supported by the Fundamental Research Funds for the Central Universities (Grant No. HIT.NSRIF.2015027) and the Fundamental Research Funds for the Central Universities (Grant No. HIT.KLOF.2013)

\section{References}

[1] Noor A.K., Recent advances and applications of reduction methods, Applied Mechanics Reviews 47, 125-146(1994).

[2] Nair P.B. et al., Improved first-order approximation of eigenvalues and eigenvectors, AIAA Journal 36, 1712-1727(1998).

[3] Aktas E and Moses F, Reduced basis eigenvalue solutions for damaged structures, Mechanics of Structures and Machines 26, 63-79.

[4] Kirsch U, Improved stiffness-based first-order approximation for structural optimization, AIAA Journal 33, 143-150(1995).

[5] Kirsch U, Approximate vibration reanalysis of structures, AIAA Journal 41, 504-511(2003).

[6] Kirsch U, Bogomolni M. Error evaluation in approximate reanalysis of structures, Structural and Multidisciplinary Optimization 28(2-3), 77-86 (2004a).

[7] Kirsch U, Bogomolni M. Procedures for approximate eigenproblem reanalysis of structures, International Journal for Numerical Methods in Engineering 60(12), 1969-1986(2004b).

[8] He J.J., Jiang J.S. and $\mathrm{Xu} \mathrm{B,} \mathrm{Modal} \mathrm{reanalysis} \mathrm{methods} \mathrm{for} \mathrm{structural} \mathrm{large}$ topological modifications with added degrees of freedom and non-classical damping, Finite Elements in Analysis and Design 44(1-2), 75-85(2007).

[9] Rong F, Chen S.H., Chen Y.D., Structural modal reanalysis for topological modifications with extended Kirsch method, Computer Methods in Applied Mechanics and Engineering 192(5-6), 697-707(2003).

[10] Ma L, Chen S.H. and Meng G.W. ,Combined approximation for reanalysis of complex eigenvalues, Computers and Structures 87(7-8), 502-506 (2009).

[11]Yang Z.J., Chen S.H. and Wu X.M. ,A method for modal reanalysis of topological modifications of structures, International Journal for Numerical Methods in Engineering 65(13), 2203-2220(2006).

[12]Chen S.H. and Rong F,A new method of structural modal reanalysis for topological modifications, Finite Elements in Analysis and Design 38(11), 1015-1028(2002).

[13]Zhang G, Nikolaidis E and Mourelatos Z.P.,An Efficient reanalysis methodology for probabilistic vibration of large scale structures, Journal of Mechanical Design 131(5), 1-13(2009). 
[14]Liu X.L. and Oliveira C.S., Iterative modal perturbation and reanalysis of eigenvalue problem, Communications in Numerical Methods in Engineering 19(4), 263-274(2003).

[15]Chen S.H., Wu X.M. and Yang Z.J.,Eigensolution reanalysis of modified structures using epsilon-algorithm, International Journal for Numerical Methods in Engineering 66(13), 2115-2130(2006).

[16]Chen S.H., Ma L and Meng G.W. ,Dynamic response reanalysis for modified structures under arbitrary excitation using epsilon-algorithm, Computers and Structures 86(23-24), 2095-2101(2008).

[17] Yang X.W., Chen S.H. and Wu B.S.,Eigenvalue reanalysis of structures using perturbations and Pade approximation, Mechanical Systems and Signal Processing 15(2), 257-263(2001).

[18]Chen S.H., Song D.T. and Ma A.J.,Eigensolution reanalysis of modified structures using perturbations and Raleigh quotients, Communications in Numerical Methods in Engineering 10, 111-119(1994).

[19]Chen S.H., Yang X.W. and Lian H.D.,Comparison of several eigenvalue reanalysis methods for modified structures,Structural and Multidisciplinary Optimization 20(4), 253-259(2000).

[20]William B.B.,An improved computational technique for perturbations of the generalized symmetric linear algebraic eigenvalue problem, International Journal for Numerical Methods in Engineering 24(3), 529-541(1987).

[21]Chen S.H., Song D.T. and Ma A.J.,Eigensolution reanalysis of modified structures using perturbations and Raleigh quotients, Communications in Numerical Methods in Engineering 10, 111-119(1994). 